\title{
CONHECIMENTO E PERCEPÇÃO DE IDOSOS FRENTE A PANDEMIA DA
}

\section{COVID-19}

Vitória Nazaré Moreira Gomes Araújo; Universidade do Estado do Pará (UEPA); vitoria.araujo07@outlook.com Fredson Murilo de Oliveira Teixeira; Universidade do Estado do Pará (UEPA); fredson.murilo31@gmail.com

Nicole Salomão Lopes; Universidade do Estado do Pará (UEPA); nicolesalomaol@gmail.com João Vitor Tavares Carneiro; Universidade do Estado do Pará (UEPA); jvitortavaresc@gmail.com Josué Bengtson Netto; Universidade do Estado do Pará (UEPA); josuebengtsonnetto@gmail.com

Brenda Nazaré Gomes Andriolo; Grupo Cynthia Charone; nep@hospitalcynthiacharone.com Niele Silva de Moraes; Grupo Cynthia Charone; nielemoraes@yahoo.com.br Cynthia Cyllene de Oliveira Charone; Grupo Cynthia Charone; cynthiacharone@yahoo.com.br

\section{RESUMO}

Introdução: A pandemia do novo coronavírus (SARS-CoV-2) trouxe um grande impacto para a saúde, não só física, mas também mental da população em todo o mundo, afetada tanto pelo isolamento quanto pela preocupação com o adoecimento. Ademais, trouxe a demanda de orientações de prevenção, sobretudo para a população geriátrica, considerada grupo de risco. Objetivo: Estimar o nível de conhecimento e a percepção sobre o novo coronavírus em idosos residentes da região metropolitana de Belém durante a pandemia da COVID-19. Métodos: Trata-se de um estudo transversal realizado mediante monitoramento a distância e com protocolo próprio nos meses de abril e maio de 2020, sendo a amostra composta por pessoas com 60 anos ou mais que concordaram em participar. A pesquisa foi realizada no Grupo Cynthia Charone e os dados foram analisados por estatística descritiva. Resultados: Dos 758 idosos avaliados, 96,6\% declararam ter conhecimento sobre o novo coronavírus. Em relação ao nível de preocupação acerca da pandemia, 65,8\% afirmaram estar muito preocupados, $22,4 \%$ pouco preocupados e 11,8\% não apresentavam preocupação. Ainda, 94,6\% dos idosos relataram saber como se prevenir da doença. Por fim, os meios mais utilizados para obtenção de informações sobre a prevenção foram, respectivamente, a mídia, a família/os amigos, o serviço e outros profissionais da saúde. Conclusão: Assim, observam-se altos índices de conhecimento e preocupação acerca da COVID-19. Destaca-se também o papel da mídia na divulgação de informações sobre prevenção da pandemia, democratizando a informação e criando uma cultura de cuidados para o combate da infecção.

Palavras-chave: Saúde do idoso; Coronavirus; Pandemia. 